\title{
COULOMETRIC SENSORS, THE APPLICATION OF A SENSOR- ACTUATOR SYSTEM FOR LONG-TERM STABILITY IN CHEMICAL SENSING*
}

\section{BART VAN DER SCHOO'T and PIET BERGVELD}

Department of Electrical Engineering, Twente University of Technology, P.O. Box 217 , 7500 AE Enschede (The Netherlands)

(Received November 13, 1986; accepted September 1, 1987)

\section{Abstract}

We present a new type of chemical transducer, the coulometric sensor. This sensor is in fact an integrated sensor-actuator system that is able to measure the concentration of acids and bases by means of a coulometric titration. An ISFET is used as the sensor to monitor the pH changes induced by the actuator, which is a gold electrode that fits closely around the ISFET's gate area. Coulometry is an absolute method and therefore the output of the new sensor is only determined by its dimensions and is not subject to changes in offset and sensitivity of the indicator electrode. It is thus expected that the operation of the sensor will be stable for a long time, so that only a onetime calibration is needed. As a first example of this new class of chemical transducers, a carbon dioxide sensor is presented. It is shown that the stability is some orders of magnitude better than that for a 'classical' potentiometric sensor.

\section{Introduction}

Direct potentiometric measurement of ion concentrations is a powerful method and is widely used. Because of the high speed of the direct method, it is very useful for continuous monitoring. However, potentiometric methods require a rigid control over experimental variables such as temperature and ionic strength if accurate measurements are to be made. If, for example, a $1 \mathrm{mV}$ error is made in the reading of the electrode potential for the detection of a univalent ion, this will result in a $4 \%$ error in concentration, as can be seen from the logarithmic term in the Nernst equation. For divalent ions this error will even be doubled. It will be clear that any drift in the potential of the ion-sensitive electrode or in the potential of the reference

* Based on a paper presented at the 2nd International Meeting on Chemical Sensors, Bordeaux, France, July 7 - 10, 1986. 
electrode will, after some time, result in smaller or larger errors. Therefore frequent recalibration of these systems is a necessity.

In this respect, ion-sensitive field effect transistors (ISFETs) are not different from any other type of ion-selective electrode. For measurements over a short period, a correction can sometimes be applied if the drift characteristics of the electrode are known and the sensitivity does not change [1]. For long-term applications, however, this method will not hold and some kind of calibration has to be applied. Bergveld and Koning [2] suggested an in vivo calibration method through the occasional injection of small amounts of buffer solution along the $\mathrm{pH}$-sensitive area of an ISFET located in the tip of an indwelling catheter. Apart from the ethical problems that this approach might give, it is of course limited to catheter-type sensors for temporary use. On the other hand, the method is of course applicable to any kind of sensor and is not limited to $\mathrm{pH}$ determinations. For extracorporeal use, Sibbald et al. [3] developed a four-function ChemFET integrated circuit that can be checked regularly with standard calibration solutins. For in vivo calibration of sensors this procedure with a number of standard solutions is impractical. We therefore suggest a different approach.

\section{Coulometric sensors}

In a classical chemical sensor, information flows only in one direction. The sensor converts the concentration of a chemical species into a voltage or a current, which is then fed into some sort of measuring instrument. In this paper, we present the integration of a chemical sensor with a corresponding actuator, as shown in Fig. 1. Now information not only flows from the chemical environment to the data processing system, but also the other way around. The actuator can actually change the composition of the chemical environment and if the relationship between the actuator input and the resulting change in $\mathrm{pH}$ is known, the sensor can be tested.

In practice, the influence of the actuator output must, of course, be limited to an ultimately small fraction of the chemical environment. Therefore, the sensor-actuator system should contain a small cavity that is separated from the environment by some sort of membrane.

The $\mathrm{pH}$ actuator used for the work described here is of the coulometric type. In a previous paper [4] we have shown that coulometry performed in nanolitre volumes provides useful information on the composition of a solution very rapidly.

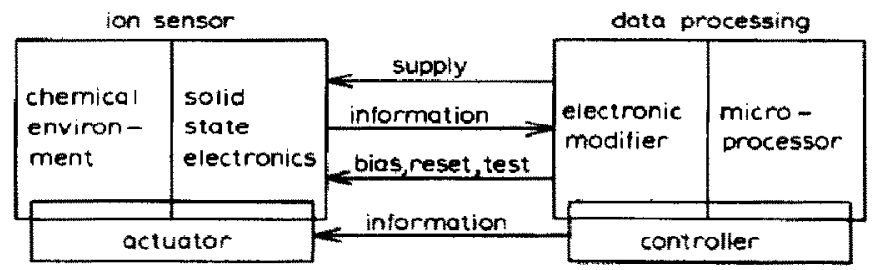

Fig. 1. Block diagram of a chemical sensor-actuator system. 


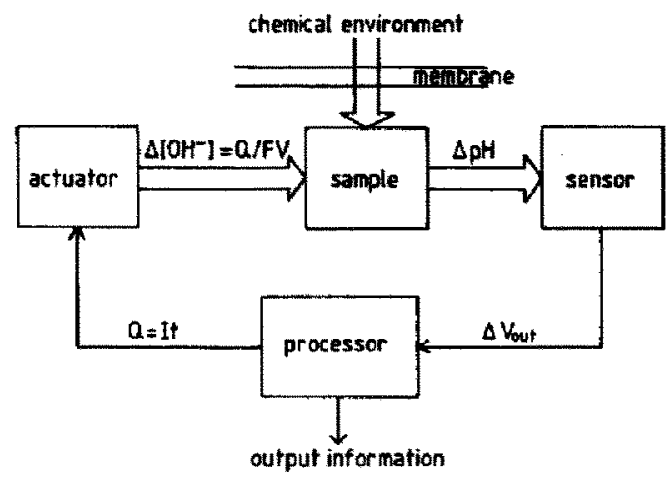

Fig. 2. Block diagram of a coulometric sensor. An amount of charge $Q$, which is equal to the generating current $I$ times the pulse width $t$, is converted by the actuator. This results in a change in [OH ] equal to $Q / F V$ in which $F$ is Faraday's constant and $V$ is the sample volume. Through the change in $\mathrm{pH}$, a variation in the output voltage of the sensor is created, which is measured by the processor.

The system, as depicted in Fig. 2, consists basically of a pH-sensitive ISFET and a set of noble-metal electrodes for coulometric generation, enclosed in a cavity in which the sample is held in place by a membrane. We will call this structure a coulometric sensor. It is through the feedback of information that this sensor should have improved stability and be more suitable for long-term use.

The coulometric sensor may be used for two purposes. First, the output from the actuator can be a test signal for the ISFET, which may be calibrated in this way. This procedure can be repeated every now and then and the ISFET can be used in a normal way as a potentiometric electrode. The second possibility is to use the actuator to measure the concentration of certain constituents in the sample and use the ISFET only as an indicator electrode for the progress of a coulometric titration. The distinction between the two methods is not absolute. The operation of the actuator will always depend on the properties of the sample and thus the first method also yields some information on its composition. The major difference is that with the first method, the ISFET is used in a normal potentiometric way in between calibrations and therefore requires some sort of stable reference electrode. With the second method the information is obtained from a measurement of relatively large changes in $\mathrm{pH}$ within a short time, and thus the requirements for the reference electrode are less stringent.

Choice of the membrane

The operation of a coulometric sensor will depend on the nature of the membrane that is used to separate the sample from the environment in which the measurement is to be carried out. One possibility is the use of a neutral membrane, e.g., a dialysis membrane that is permeable for all constituents of the solution up to a certain size. In that case the titration volume will contain most of the buffering components that are available in the bulk solution 
with the exception of, for instance, proteins. (The exclusion of the last is possibly of influence on the result of the measurement but, on the other hand, fouling of the generating electrodes may be prevented in this way.) If the bulk solution only contains a single buffering component, the analysis of the titration data will not cause great problems [4]. However, should this type of sensor be used for, e.g., biomedical purposes such as a measurement in blood, the sample solution will be more complex and, next to phosphate and carbonate, will also contain a number of organic acids [5]. In that case the titration curves that are obtained will be more difficult to interpret. It must be noted that a permselective membrane, e.g., a membrane only permeable to cations or anions, cannot be used here. A potential difference will develop across such a membrane as a function of concentration and this will prevent further transport. Thus the concentration of a specific ion will not necessarily become the same on both sides of the membrane.

Another possibility is to choose a certain ionic composition of the sample solution and use a membrane that is not permeable for ions but only for volatile components. When a gas-permeable membrane is used to enclose the sample solution, the coulometric sensor is in fact a Severinghaus electrode [6] that is sensitive to, for instance, carbon dioxide. The operation of the coulometric sensor is now determined by only one buffering component, i.e., carbonic acid. In this paper we will describe the development of such a coulometric carbon dioxide sensor. The realization of a self-calibrating sensor for $\mathrm{pH}$, instead of $\mathrm{CO}_{2}$, is a more complex task and is currently under investigation in our laboratory.

Construction of the coulometric sensor

The carbon dioxide sensor presented here is based on the coulometric analyser that was described earlier [4]. It consists of an experimental $9.5 \times$ $19 \mathrm{~mm}^{2}$ silicon chip containing ten ISFETs. On the chip, four gold electrodes are deposited for coulometric generation. On top of this chip a silicon cover is sealed with two holes in it through which the sample solution can be introduced. To convert this structure into a carbon dioxide sensor, a third hole, measuring $1 \times 2 \mathrm{~mm}^{2}$, is etched in the cover. This hole is closed with a Teflon membrane $3 \mu \mathrm{m}$ thick. A spacer is placed between this cover and the ISFET chip, thus creating a gap of approximately $30 \mu \mathrm{m}$ for the inner electrolyte layer. The electrolyte can be introduced through the remaining two holes in the cover. A cross-section of the sensor is shown in Fig. 3.

When the system is filled with a bicarbonate solution, it can be used like a normal Severinghaus electrode. In this case an external reference electrode is used connected to the sensor via a tube to one of its filling holes. The fabrication of a silver/silver chloride reference on the same chip is of course possible [7], but at this moment not of special interest to us. The response of the system is tested by exposing it to humidified gas streams of different $\mathrm{CO}_{2}$ concentration. Figure 4 shows the change in the output voltage 

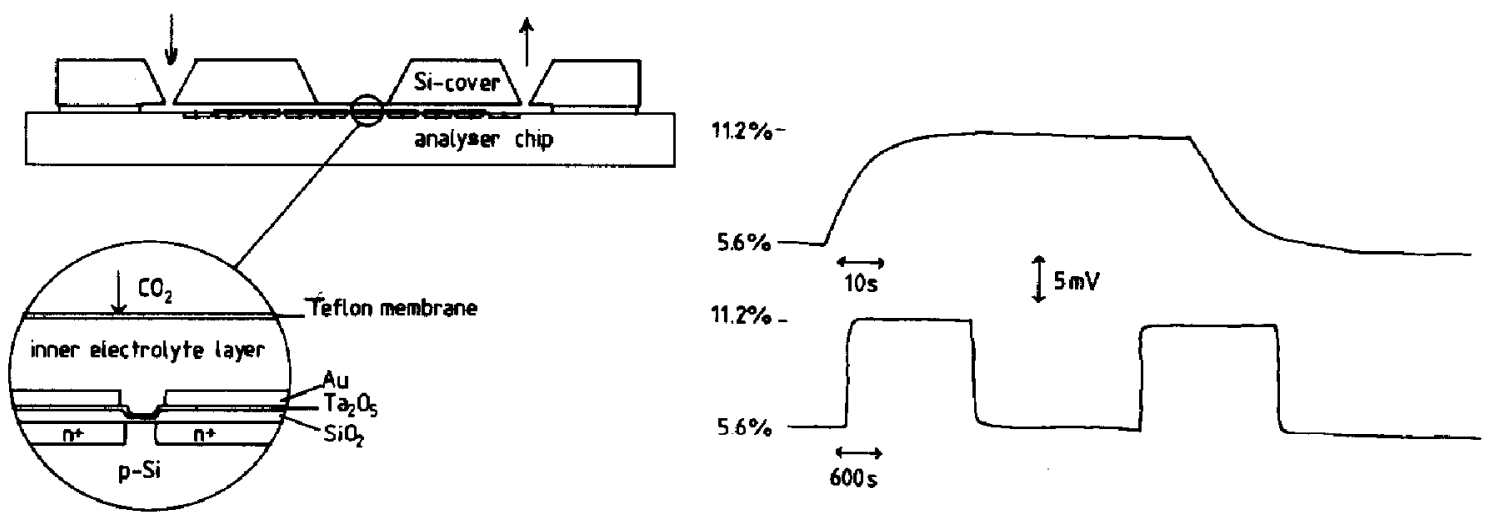

Fig. 3. Cross-section of the coulometric carbon dioxide sensor.

Fig. 4. Dynamic response of the carbon dioxide sensor used as a normal Severinghaus electrode, on a time scale of minutes and hours.

as a result of a stepwise change in concentration. When the concentration of $\mathrm{CO}_{2}$ is doubled, a corresponding $\mathrm{pH}$ change of 0.3 units can be expected. The pH sensitivity of the ISFETs used was $43 \mathrm{mV} /$ decade, and from the Figure it can be seen that the sensitivity to $\mathrm{CO}_{2}$ is practically equal to the theoretical value. The time constants found in this case are in good agreement with the theoretical predictions made elsewhere [8].

In a coulometric sensor, $\mathrm{CO}_{2}$ is not detected from the $\mathrm{pH}$ value of the inner solution but by an acid-base titration performed in this solution. Although the speed of hydration of $\mathrm{CO}_{2}$ is slow, it does not influence the response of a Severinghaus type of electrode [8]. When a rapid titration of $\mathrm{CO}_{2}$ is attempted, however, the hydration speed does become a limiting factor because the total amount of carbon dioxide has to be converted into bicarbonate in this case. This problem will be addressed in the next section.

\section{Titration of carbon dioxide}

Carbon dioxide in an aqueous solution behaves like a weak acid ( $\mathrm{p} K_{\mathrm{a}}=$ 6.34). This acidic reaction is the result of a hydration process in which carbonic acid is formed:

$\mathrm{CO}_{2}+\mathrm{H}_{2} \mathrm{O} \rightleftharpoons \mathrm{H}_{2} \mathrm{CO}_{3} \rightleftharpoons \mathrm{H}^{+}+\mathrm{HCO}_{3}^{-}$

At equilibrium conditions the ratio $\left[\mathrm{CO}_{2}\right] /\left[\mathrm{H}_{2} \mathrm{CO}_{3}\right] \approx 600$. When the solution is titrated with hydroxyl ions, two reactions occur. The first is the conversion of carbonic acid to bicarbonate:

$\mathrm{H}_{2} \mathrm{CO}_{3}+\mathrm{OH}^{-} \rightleftharpoons \mathrm{HCO}_{3}^{-}+\mathrm{H}_{2} \mathrm{O}$

A second possibility is the direct reaction between $\mathrm{CO}_{2}$ and $\mathrm{OH}^{-3}$ :

$\mathrm{CO}_{2}+\mathrm{OH}^{-} \rightleftharpoons \mathrm{HCO}_{3}^{-}$ 
Because both reactions (1) and (3) are rather slow [9], problems arise when a titration has to be performed within a number of seconds. The hydration reaction (1) is a pseudo first-order process:

$-\mathrm{d}\left[\mathrm{CO}_{2}\right] / \mathrm{d} t=k_{\mathrm{CO}_{2}}\left[\mathrm{CO}_{2}\right]$

The value of $k_{\mathrm{CO}_{2}}$ is $0.03 \mathrm{~s}^{-1}$, which means that the process has a time constant of approximately $30 \mathrm{~s}$. The direct reaction (3) is a second-order process:

$-\mathrm{d}\left[\mathrm{CO}_{2}\right] / \mathrm{d} t=k_{\mathrm{OH}}\left[\mathrm{CO}_{2}\right]\left[\mathrm{OH}^{-}\right]$

It can be seen from this equation that the reaction speed is $\mathrm{pH}$ dependent. $k_{\mathrm{OH}}=8500 \mathrm{~s}^{-1}(\mathrm{~mol} / \mathrm{l})^{-1}$ and therefore the process is only relatively fast at high $\mathrm{pH}$ values; for example at $\mathrm{pH}=10$, the time constant $\tau=1.2 \mathrm{~s}$ and at $\mathrm{pH}=11, \tau=0.12 \mathrm{~s}$. From these values it can be seen that a direct titration of carbon dioxide is not possible within a reasonably short time. In Fig. 5, the calculated titration curves for a $0.002 \mathrm{M}$ solution of carbon dioxide are shown for various titration speeds. Because only a small amount of the total $\mathrm{CO}_{2}$ is available as $\mathrm{H}_{2} \mathrm{CO}_{3}$ to give a fast buffering action, at the higher titration speeds very soon an excess of free $\mathrm{OH}^{-}$ions will be formed and the $\mathrm{pH}$ will rise quickly. To obtain a 'normal' curve as drawn for the infinitely slow titration $(\infty)$, from which a useful equivalence point can be detected, the titration time should be at least of the order of five minutes. It will be clear that this is rather long for application in a sensor.

The problem can be solved by performing a back titration. First, an amount of hydroxyl ions in excess of the expected $\mathrm{CO}_{2}$ concentration is quickly added to the solution. The $\mathrm{pH}$ will thus be raised to a value between $\mathrm{pH} 10$ and $\mathrm{pH}$ 11. At this high $\mathrm{pH}$ value, the direct reaction between $\mathrm{CO}_{2}$ and $\mathrm{OH}^{-}$proceeds at relatively high speed (eqn. (5)). The conversion of $\mathrm{CO}_{2}$ will be completed within some seconds, while part of the generated hydroxyl ions will be consumed by the reaction. The excess of hydroxyl ions can now be titrated by the generation of hydrogen ions at a sufficiently high speed and the original $\mathrm{CO}_{2}$ concentration can be determined. Figure 6 shows the simulated titration of different $\mathrm{CO}_{2}$ concentrations followed by the back titration of the excess $\mathrm{OH}^{-} .9 \mathrm{mmol} / 1$ of $\mathrm{OH}^{-}$is added over a period of $4 \mathrm{~s}$; after a pause of $1 \mathrm{~s}$ the same amount of $\mathrm{H}^{+}$is supplied. While the forward titration reveals hardly any information about the concentration of carbon dioxide, a clear shift in the position of the equivalence point can be seen during the back titration. It should be noted that for the calculation of Figs. 5 and 6 , it is assumed that the solution is perfectly mixed so that only the reaction kinetics are studied.

\section{Operation of the coulometric sensor}

The analyser chip contains four gold electrodes. Coulometric titration is performed by using one of these as the working electrode to produce hydroxyl ions or protons. In order to obtain a homogeneously distributed 

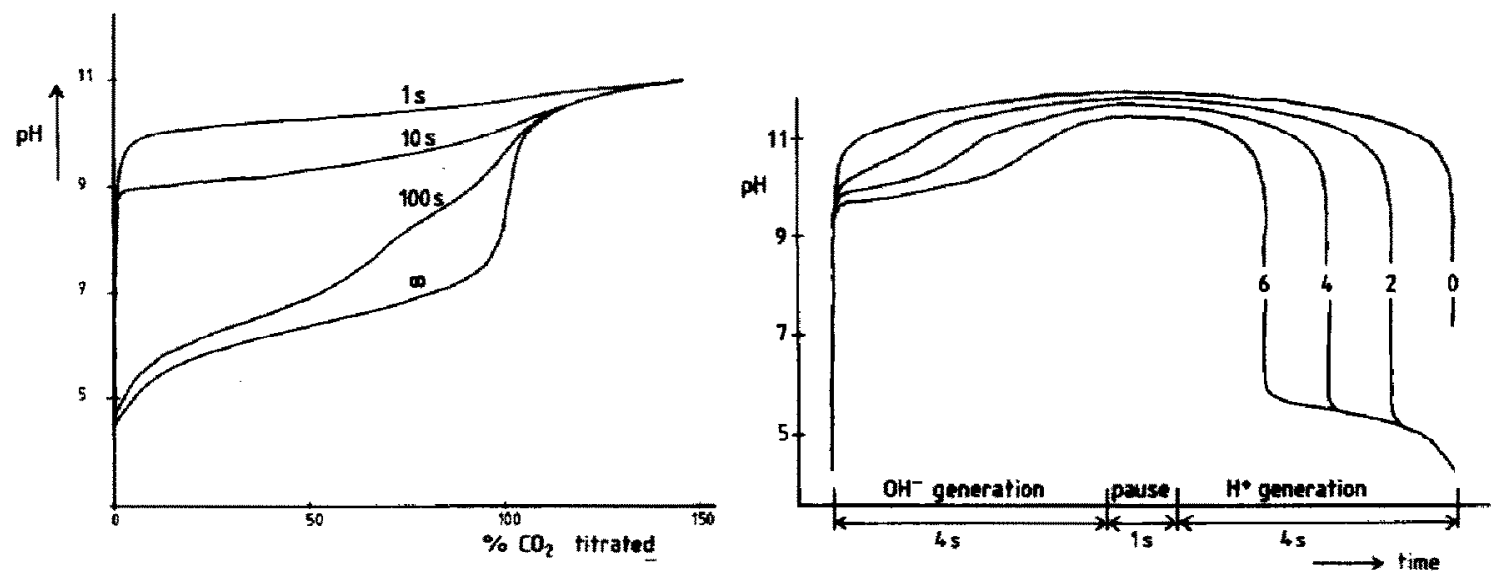

Fig. 5. Simulated titration curves for $0.002 \mathrm{M}$ carbon dioxide in a perfectly mixed solution at various titration speeds. An equivalent amount of $\mathrm{OH}^{-}$ions is added in the time indicated on the curves.

Fig. 6. Back titration of the excess of $\mathrm{OH}^{-}$ions after the titration of $\mathrm{CO}_{2}$ in a perfectly mixed solution. The figures on the curves indicate the original $\mathrm{CO}_{2}$ concentration in mmol/l.

generation of ions across this electrode, two counter electrodes are used, one on each side of the working electrode. The fourth gold electrode is used as a pseudo reference electrode. Because only local $\mathrm{pH}$ changes are generated during a titration experiment, a normally $\mathrm{pH}$-sensitive ISFET located at a point of the inner electrolyte layer at which the $\mathrm{pH}$ is not affected can be used as a reference. A differential measurement is performed using one ISFET at the site of the titration and one at this so-called neutral site. Both ISFETs measure with respect to the pseudo reference gold electrode and thus any instabilities in the potential of this electrode will be cancelled out by the differential measurement. Through this procedure the use of a 'real' reference electrode becomes unnecessary, which reduces technological problems in the fabrication of these sensors.

The operation of the coulometric sensor may be best explained on the basis of a typical output registration. Figure 7 shows the titration of carbon

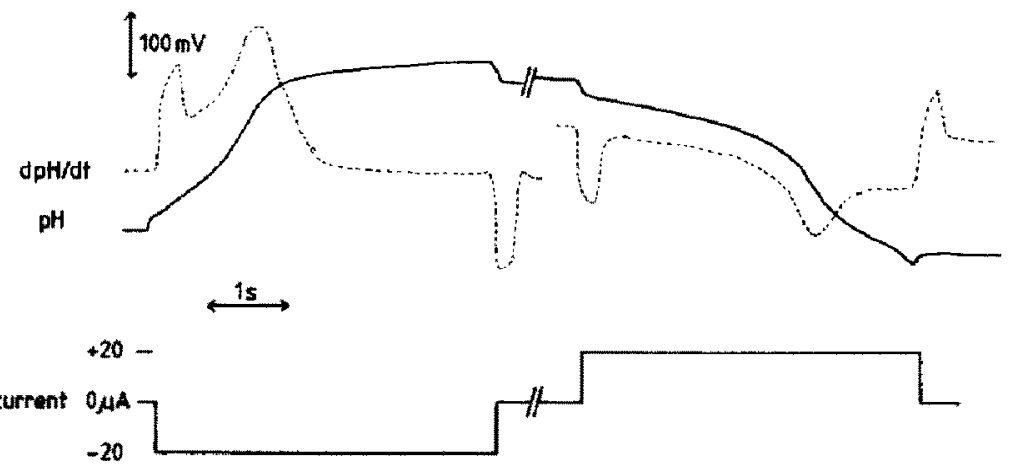

Fig. 7. Titration of $0.004 \mathrm{M}$ carbon dioxide. The equivalence points can easily be detected from the first derivative, $\mathrm{dpH} / \mathrm{d} t$. 
dioxide. Hydroxyl ions are generated for $4 \mathrm{~s}$ and after a subsequent pause of $4 \mathrm{~s}$, the current direction is reversed and hydrogen ions are generated, also for $4 \mathrm{~s}$. The titration of carbon dioxide is complicated for two reasons. The first is the slow kinetics, as we have seen in the previous section, and the second is diffusion of $\mathrm{CO}_{2}$ through the membrane during the titration. In the first phase a rapid increase in $\mathrm{pH}$ can be seen and an equivalence point is reached within $1.5 \mathrm{~s}$. Only a small fraction of the total $\mathrm{CO}_{2}$ is available as carbonic acid to give a fast buffering reaction, and therefore an excess of free hydroxyl ions is quickly formed. Now two things will happen. At the high $\mathrm{pH}$ value that is soon reached, $\mathrm{CO}_{2}$ will react directly with $\mathrm{OH}^{-}$according to eqns. (3) and (5). Because the time constant of the reaction is atill of the order of 0.1 to $1 \mathrm{~s}$, some time is needed between the generation of $\mathrm{OH}^{-}$ and the back titration to allow the conversion of carbon dioxide to be completed. If this pause is too short, the $\mathrm{CO}_{2}$ concentration will be virtually lowered and the second equivalence point will shift to the right. On the other hand, as $\mathrm{CO}_{2}$ in the inner electrolyte layer is consumed, it will be replenished through the membrane. This effect increases the total amount of $\mathrm{CO}_{2}$ and causes the equivalence point to shift to the left. This effect will become less important when the membrane thickness is increased and diffusion is thus slowed down. However, a thicker membrane will lead to an increased response time to a change in the outside $\mathrm{CO}_{2}$ concentration. Thus the membrane thickness is an important parameter in the operation of the coulometric sensor and has to be optimized.

When the simulation in Fig. 6 and the experimental curve in Fig. 7 are compared, some differences are obeserved. In Fig. 6, only the reaction kinetics are taken into account. From Fig. 7 it is clear that the result is not only determined by the slow kinetics but also by diffusion of ions. Because it is impossible to locate the generating electrode and the pH-sensing ISFET at exactly the same place, some time lag is observed between the beginning of the current pulse and the occurrence of the equivalence point in the forward titration. This subject is addressed in more detail in ref. 10.

For the generation of hydroxyl ions and protons, quinhydrone is added to the inner electrolyte solution of the sensor. Quinhydrone, which is a 1:1 mixture of $p$-benzoquinone and hydroquinone, is oxidized and reduced at much lower electrode potentials than those required for the electrolysis of water [4]. The electrode reactions that take place are:

$p$-benzoquinone $+2 \mathrm{H}^{+}+2 e \rightleftharpoons$ hydroquinone

Although the addition of quinhydrone facilitates the generation of reproducible $\mathrm{pH}$ changes, it has the disadvantage that hydroquinone is a weak acid $\left(\mathrm{p} K_{\mathrm{a}}=10.47\right)$. Therefore, since an excess of quinhydrone is required, the solution becomes effectively buffered at a $\mathrm{pH}$ value of approximately $\mathrm{pH}=10.5$ when hydroxyl ions are generated. It will be clear that the speed of conversion of carbon dioxide to bicarbonate ions is thus somewhat limited at this $\mathrm{pH}$. 
Experimental results

The performance of the sensor is tested by exposing it to humidified gas streams of different $\mathrm{CO}_{2}$ concentration. The concentration in this sample stream is measured with an $\mathbf{E 5 0 3 6} \mathrm{pCO}_{2}$ electrode from Radiometer Copenhagen and this electrode is calibrated agaisnt two standard $\mathrm{CO}_{2}$ concentrations from a GMA2 precision gas supply, also from Radiometer.

The excess of hydroxyl ions that is titrated depends first of all on the original $\mathrm{CO}_{2}$ concentration. Furthermore, the total length of the procedure affects the titration time due to the slow conversion of $\mathrm{CO}_{2}$ into bicarbonate and the diffusion of extra $\mathrm{CO}_{2}$ through the membrane as mentioned before. In ref. 10, some consideration is given to the selection of the proper procedure in relation to the diffusion parameters and the reaction kinetics. Figure 8 shows the results for two different titration protocols. The generation current is $20 \mu \mathrm{A}$ in both cases. The length of the generation period of hydroxyl ions and protons is $4 \mathrm{~s}$. The pause between base and acid generation, however, is $1 \mathrm{~s}$ in the first case (4-1-4) and $4 \mathrm{~s}$ in the other (4-4-4). The time needed for the back titration depends on the concentration in both cases. If the pause length is increased, the amount of $\mathrm{CO}_{2}$ that takes part in the reaction also increases due to the effects discussed in the previous section. The third line in Fig. 8 shows the results of the forward titration in the first phase. This phase is equal for both titration protocols. It can be seen that the influence of the carbon dioxide concentration on the time needed to reach the first equivalence point is negligible.

It will be clear that the sensor has to be calibrated for each different titration protocol. Once this calibration has been performed, however, it is stable for a long period. Figure 9 shows the sensor output for a $\mathrm{CO}_{2}$ concentration over a period of 51 days, which was the lifetime of this particular coulometric sensor. On one day, the repeatability of the measurements is approximately within $10 \%$ in concentration. Over a period of seven weeks,

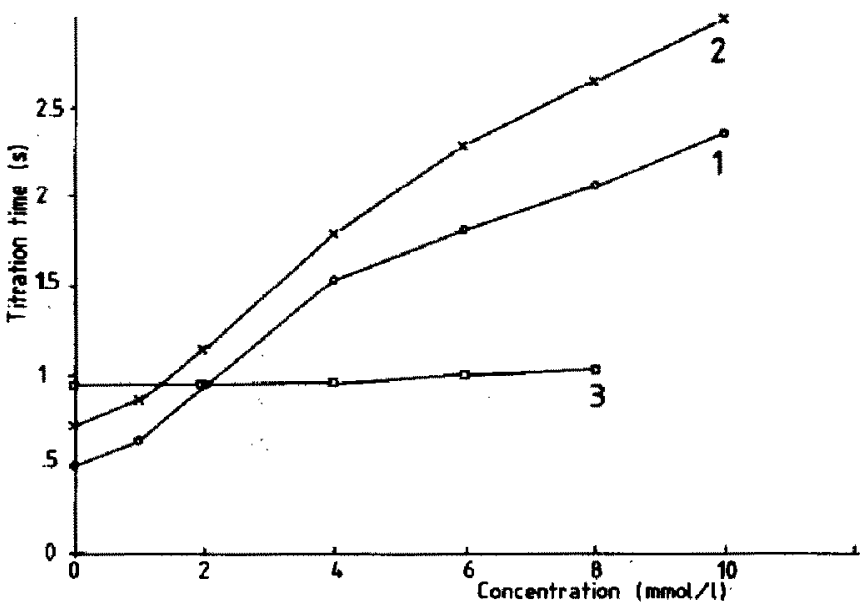

Fig. 8. Times needed for the back titration as a function of concentration for two protocols, 4-1-4 (1) and 4-4-4 (2), compared to the forward titration (3). 


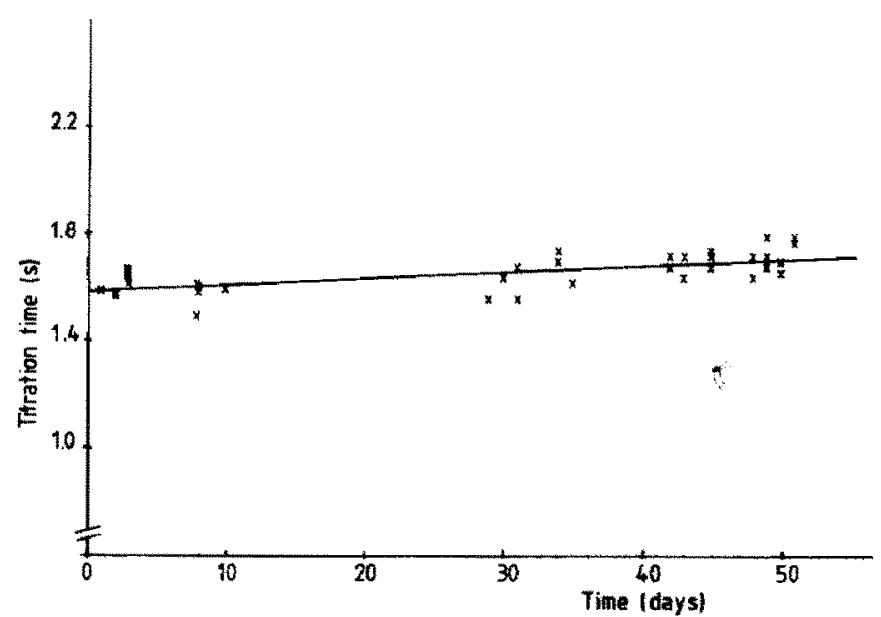

Fig. 9. Sensor output during its lifetime for a $\mathrm{CO}_{2}$ concentration of $0.004 \mathrm{~mol} / \mathrm{l}$.

the average titration time for this concentration increases, also with approximately $10 \%$ in concentration.

\section{Discussion}

The first results of the coulometric sensor have been presented here. We have shown this new type of electrochemical sensor to be a working concept. Future work should concentrate on the optimization of the applied materials and the dimensions of the sensor.

The first measurements show a sensor drift of approximately $10 \%$ in concentration over a period of seven weeks. Compared to a potentiometric sensor with a logarithmic output, this comes down to a total drift of $2.5 \mathrm{mV}$ or an average of $0.05 \mathrm{mV} /$ day. The drift that can be expected for a 'normal' ISFET-based potentiometric sensor is at least of the order of $0.1-1 \mathrm{mV} / \mathrm{h}$. At this moment, the precision of the measurements is not better than within $\pm 10 \%$. For biomedical applications, this should be improved at least by a factor of two [11].

The carbon dioxide concentrations is measured by the reverse titration of an excess of hydroxyl ions that is added to the solution. To make an accurate determination, the amount of hydroxyl ions that is generated initially must be in reasonable proportion to the expected carbon dioxide concentration. Thus, for the selection of the right titration protocol, knowledge of the approximate concentration is required. This, of course, can be obtained from a first experiment in which the concentration is roughly estimated. In a second experiment, the measurement can then be repeated, using the optimal protocol. In this way the dynamic range of the coulometric sensor might be expanded. So far we have only considered one decade in concentration.

Coulometry is an absolute method of measurement and the sensor measures the amount of $\mathrm{OH}^{-}$ions needed for the conversion of an amount 
of acid. Because the equivalence point is detected from the first derivative of the titration curve, offset and sensitivity of the indicator ISFET are not important. The behaviour of the coulometric sensor is determined by diffusion of ions during the titration and diffusion constants will change somewhat with ionic strength and temperature. However, these effects will be far less dramatic than the influence that these parameters will have on a direct potentiometric measurement and in general, no corrections will be needed.

The measurement of $\mathrm{CO}_{2}$ forms an exception in this respect. First of all a correction has to be made for the change in solubility of $\mathrm{CO}_{2}$ as a function of temperature, which is approximately $3 \% /{ }^{\circ} \mathrm{C}$ at room temperature [12]. This correction has to be made with any kind of $\mathrm{CO}_{2}$ measurement. A problem that is specific for the coulometric sensor is that the speed of reactions (1) and (3) increases as temperature rises. Therefore, a protocol has to be chosen in which the pause between base and acid generation is long enough for the conversion of $\mathrm{CO}_{2}$ to be completed even at the lowest temperature at which the sensor is employed. The membrane thickness has to be such that diffusion through the membrane during the titration is negligible.

Of course, the sensor presented here for carbon dioxidé can, in principle, also be used for other volatile components such as $\mathrm{NH}_{3}, \mathrm{SO}_{2}, \mathrm{Cl}_{2}$ and so on [6]. Instead of a gas-permeable membrane, a porous cellulose acetate membrane that is also permeable for ions could be used. In that case, the coulometric sensor could be used for any acidic or basic substance.

The measurements need not be limited to solutions containing only one protolyte. Provided that the difference in $\mathrm{p} K_{\mathrm{a}}$ values of the components is large enough, mixtures can also be analysed in this way. Furthermore, substances having a similar $\mathrm{p} K_{\mathrm{a}}$ can also sometimes be discerned. From the experiments described above it will be clear that the actual $\mathrm{CO}_{2}$ concentration is found from the difference between the forward and the back titration. Because of a deviating reaction speed, carbon dioxide can be distinguished in a mixture of other acids.

In conclusion, it can be said that the idea of a coulometric sensor is useful. The precision of the present sensor is certainly not very spectacular and needs to be improved. Its stability, however, looks very promising and the coulometric sensor can be used for an extended period without calibration.

\section{References}

1 P. Bergueld, The development and application of FET-based biosensors, Biosensors, 2 (1986) $15-33$.

2 P. Bergveld and G. Koning, European Patent Appl. 0036171.

3 A. Sibbald, P. D. Whalley and A. K. Covington, A miniature flow through cell with a four-function chemfet integrated circuit for simultaneous measurements of potassium, hydrogen, calcium and sodium ions, Anal. Chim. Acta, 159 (1984) 47 - 62.

4 B. H. van der Schoot and P. Bergveld, An ISFET-based microlitre titrator: integration of a chemical sensor-actuator system, Sensors and Actuators, 8 (1985) $11-22$. 
5 E. E. Selkurt, in E. E. Selkurt (ed.), Physiology, Little, Brown and Company, Boston, MA, 4th edn., 1976, Ch. 24.

6 J. W. Ross, J. H. Riseman and J. A. Krueger, Potentiometric gas sensing electrodes, Pure Appl. Chem., 35 (1973) 473 - 487.

7 L. J. Bousse, P. Bergveld and H. J. M. Geeraedts, Properties of Ag/AgCl electrodes fabricated with IC-compatible technologies, Sensors and Actuator,, 9 (1986) 179 197.

8 B. H. van der Schoot and P. Bergueld, Prediction of the dynamic response of the potentiometric carbon dioxide electrode, Anal. Chim. Acta, 166 (1984) 93 - 101.

9 D. M. Kern, The hydration of carbon dioxide, J. Chem. Educ., 37 (1960) 14 - 23.

10 B. H. van der Schoot, Coulometric sensors, the integration of a chemical seneoractuator system, Thesis, Twente University of Technology, 1986.

11 J. L. Clausen and K. M. Murray, Clinical applications of arterial blood gases, how much accuracy do we need, J. Med. Technol., 2/1 (1985) $19-21$.

12 J. H. Perry and C. H. Chilton (eds.), Chemical Engineers Handbook, McGraw-Hill, New York, 5th edn., 1973, Table 3 - 121. 